\title{
Improving patient engagement, adherence, and satisfaction in lung cancer surgery with implementation of a mobile device platform for patient reported outcomes
}

\author{
Peter J. Kneuertz, Niveditha Jagadesh, Alicia Perkins, Morgan Fitzgerald, Susan D. Moffatt-Bruce, \\ Robert E. Merritt, Desmond M. D'Souza \\ Division of Thoracic Surgery, Department of Surgery, The Ohio State University Medical Center, Columbus, Ohio, USA \\ Contributions: (I) Conception and design: DM D'Souza; (II) Administrative support: M Fitzgerald, A Perkins; (III) Provision of study materials or \\ patients: DM D'Souza, A Perkins; (IV) Collection and assembly of data: DM D'Souza, A Perkins; (V) Data analysis and interpretation: PJ Kneuertz, \\ DM D'Souza; (VI) Manuscript writing: All authors; (VII) Final approval of manuscript: All authors. \\ Correspondence to: Desmond M. D'Souza, MD, FACS. Assistant Professor of Surgery, Division of Thoracic Surgery, Department of Surgery, The \\ Ohio State University Medical Center, Columbus, Ohio, USA. Email: desmond.d'souza@osumc.edu.
}

\begin{abstract}
Background: Active patient engagement may improve their perioperative experience and outcomes. We sought to evaluate the use of a mobile device application (App) for patient engagement and patient reported outcomes (PROs) assessment following robotic lung cancer surgery.

Methods: Patients with suspected lung cancer undergoing robotic resection between January-May 2019, were offered the SeamlessMD App, which was customized to meet requirements of the thoracic enhanced recovery pathway. The App guided patients through preoperative preparation, in-hospital recovery, and postop discharge care with personalized reminders, task lists, education, progress tracking, and surveys.

Results: Fifty patients participated in the study (22.1\%). Of the 50 patients, 20 (40\%) patients completed the preoperative compliance survey, and 31 (62\%) completed the hospital satisfaction survey. A total of 62 inpatient recovery checks were completed, identifying non-compliance with incentive spirometer use in 2 (3.2\%) and patient worries about self-care after discharge in 18 (29\%) instances. Postoperative health-checks were completed by 27 (54\%) patients with a median of 3 [0-17] completed surveys per patient. Patient reported symptom scores up to 30 days after surgery, demonstrating a significant decrease maximum pain level $(\mathrm{P}=0.002)$ and anxiety scores $(\mathrm{P}<0.001)$. The App enabled health-checks improved confidence and decreased worries in over $80 \%$ of patients. Nine patients $(40.9 \%)$ reported the health-checks helped avoid $1+$ calls and 4 (18.2\%) reported the App helped avoid 1+ visits to the hospital. Over 74\% of patients reported the App was very or extremely useful in each of the preoperative, inpatient, and post-discharge settings.

Conclusions: A mobile device platform may serve as an effective mechanism to record perioperative PROs and satisfaction while facilitating patient-provider engagement in perioperative care.
\end{abstract}

Keywords: Mobile-App; patient reported outcomes (PRO); robotic lobectomy

Submitted Dec 31, 2019. Accepted for publication Jan 26, 2020.

doi: $10.21037 /$ jtd.2020.01.23

View this article at: http://dx.doi.org/10.21037/jtd.2020.01.23

\section{Introduction}

The goal of all physicians is to provide the highest quality of care to their patients; however, the sole use of measures such as hospital length of stay and complication rates do not always provide the most holistic perspective on patient experience. Despite the similarities in surgeons' training programs, there are natural discrepancies in how physicians at different institutions manage their patients. As a result, gathering the patient's perspective on their illness, management, and recovery before and after surgery 
can provide insight into not only the effectiveness of a surgeon's craftsmanship but also into whether different medical interventions are addressing and improving patient concerns (1). Patient satisfaction in health care can be thought of as the degree to which a patient feels they received high quality care (2). Their satisfaction is likely to be higher if they believe their quality of care was higher. A patient's satisfaction regarding care is a combination of both their current health state as well as their quality of life outside of, but not independent to, medicine (3).

Implementing a patient reported outcomes (PROs) program in surgery may help to better address the morbidity and lifestyle aspect of perioperative care in a personalized manner (3). In the past, various survey tools have been used to gather PROs. However, administering and taking surveys remains time intensive for patients and providers. Most existing survey methods require extensive auditing with personnel to gather, examine, and organize data. The increasing use of mobile platforms to address this problem in PRO research have determined that mobile technology coupled to engagement features can improve patient information, compliance with treatment guidelines, and administrative efficiency (4). While such mobile platforms have been implemented in other specialties, we are not aware of any currently existing research studying its effectiveness at gathering PROs in thoracic surgery.

The objective for this study was to understand the utility of a mobile application (App) platform to engage patients whilst gathering data on patient compliance, perioperative experience and satisfaction. Patient satisfaction was further examined through measures such as the ability to reach a provider and their perspective on the usefulness of the App.

\section{Methods}

\section{Study design and patient selection}

This study was carried out as part of a quality improvement initiative to pilot an enhanced recovery after surgery (ERAS) pathway for patients undergoing robotic lobectomies between January $1^{\text {st }}, 2018$ and May 31, 2019. Adult patients aged 18 or older with diagnosed or suspected lung cancer who were scheduled for robotic lung resection were recruited during their initial preoperative clinic visit. Patient participation required patients' installation of the SeamlessMD ${ }^{\circledR}$ (Seamless Mobile Health Inc., Toronto, Canada) App on their own personal device. The App was available on both Apple and Android devices or as an internet browser application for those who could did not have access to a smartphone or tablet. Additionally, for those individuals who were not equipped to utilize a mobile application on their own, they could establish a proxy (i.e., spouse, children, etc.). The App was activated and configured with the data of surgery during the same preoperative visit. The analysis of patient reported data was approved by the local Institutional Review Board (BuckIRB-2019H0008).

\section{Mobile App functionality}

The SeamlessMD ${ }^{\circledR}$ App is a HIPAA-compliant cloud-based platform for providers to educate, engage and monitor patients throughout the pre- and postoperative period. The App includes reminders, task lists, an education library, progress tracking and surveys for collecting PROs. The content of the App was customized to match our existing thoracic surgical care pathways and clinical protocols. The Seamless $M D^{\circledR}$ App guided patients through the various phases of the enhanced recovery pathway of care including pre-operative preparation, in-hospital recovery and postoperative discharge care and were followed until 30 days post-operatively. These questions and tasks delivered by the App were personalized to each patient's needs according to their existing health status and comorbidities (i.e., smoking-related questions for smokers only). A summary of engagement and survey features of the App is presented in Figure 1. The care team had access to a provider facing dashboard to access patients' reports and analytics regarding the PROs collected. Patients were also able to contact providers through the App with questions regarding their healing process or complications, thereby facilitating open provider-patient interaction.

\section{PROs}

Patient self-reported compliance with the surgical preparation was tracked by the App preoperatively. Patient's participation during recovery in the hospital was measured by daily recovery progress checks. Postoperative health checks during recovery included pain level assessment on a $0-10$ scale. Patient satisfaction with their hospital stay was surveyed on the day of discharge. Patient's perspectives on the overall usefulness of the application was surveyed at the end of the App usage period (30 days postop). Patient perceived reduction in healthcare utilization was measured through self-reported avoidance of calls to the care team, and hospital visits. 


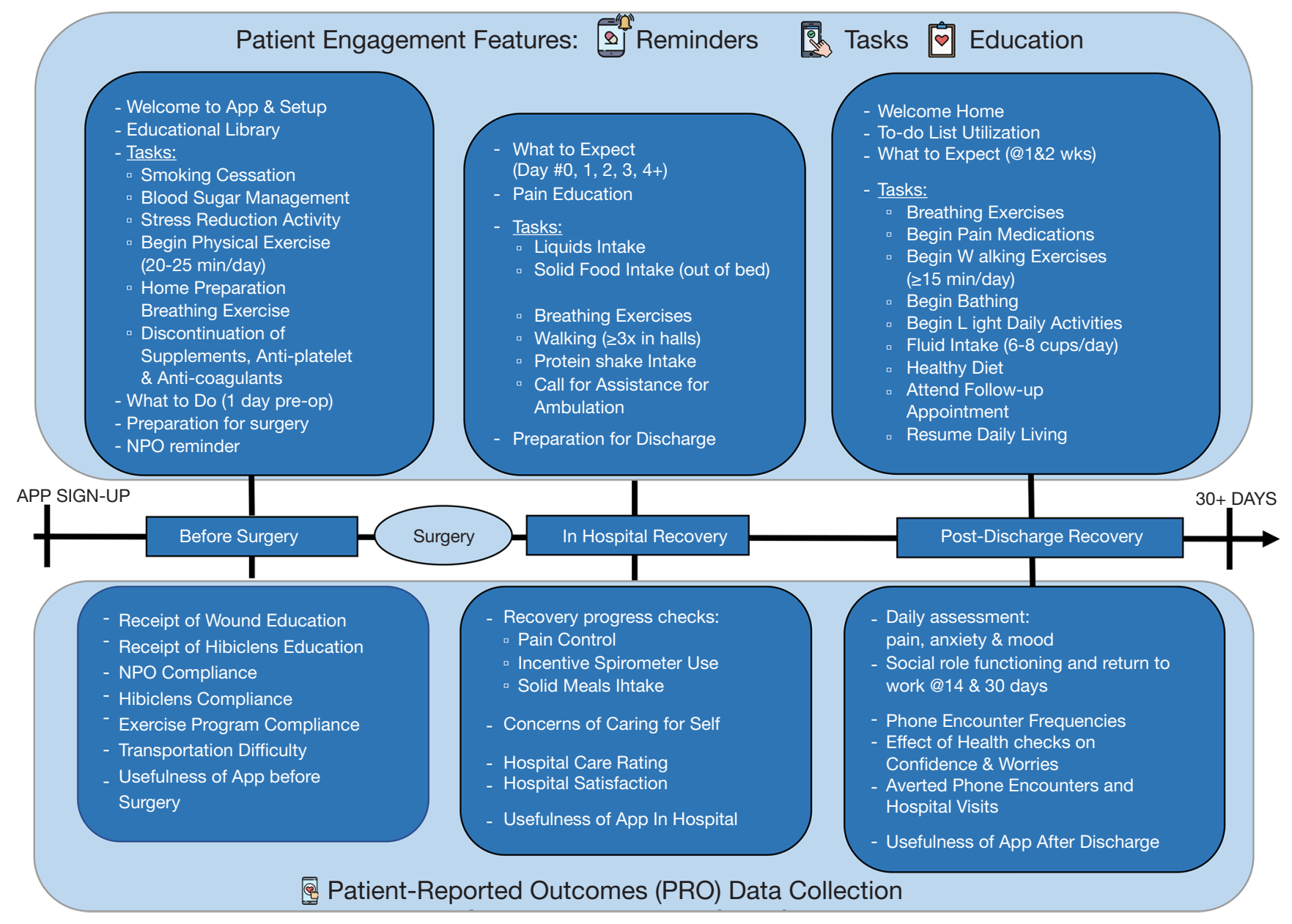

Figure 1 Overview of mobile device application enabled patient engagement and survey features.

\section{Statistical analysis}

Descriptive statistics were used to summarize patient responses in aggregate. Responses to multiple choice questions were reported as frequencies and percentages. Continuous variables were reported as medians and interquartile range (IQR). Linear mixed models with random intercepts (to account for correlated within patient measures) were used to assess the change in patient symptom scores over time after surgery. Statistical significance was defined as a $\mathrm{P}$ value $\leq 0.05$. The analysis was conducted using SAS version 9.4 (SAS Institute, Cary, NC, USA).

\section{Results}

A total of 50 (22.1\%) patients out of 226 undergoing robotic lung cancer surgery participated during the study period. Patient characteristics are summarized in Table 1. The majority of patients $(86 \%)$ underwent robotic lobectomy with an average length of stay of 3 days (Table 1).

\section{Compliance measures}

Preoperative compliance survey was returned by 20 (40\%) of patients. Almost all patients (95\%) reported having received preparative instructions on wound care and preoperative antimicrobial wash and being compliant with some or all of the preoperative exercise instructions (Table 2). Adherence to inpatient recovery instructions was tracked through patient reported daily progress evaluations. Amongst a total of 62 responses, problems were detected in $2(3 \%)$ in which patients who reported non-compliance with incentive spirometer use, 14 (23\%) reporting no 
Table 1 Demographics and clinical details

\begin{tabular}{lc}
\hline Patient characteristics & No. $(\%)(\mathrm{n}=50)$ \\
\hline Age, median $[\mathrm{IQR}]$ & $68[58,71.5]$ \\
Gender & $21(42.0)$ \\
Male & $25(50.0)$ \\
Female & $4(8.0)$ \\
Not set & $10(20.0)$ \\
Active smoker & $5(10.0)$ \\
Physical disability & $8(16.0)$ \\
Diabetes & \\
Concomitant medications & $4(8.0)$ \\
Anti-platelet & $11(22.0)$ \\
Anti-inflammatory & $2(4.0)$ \\
Warfarin & $19(38.0)$ \\
Aspirin & $2(4.0)$ \\
Anti-coagulant & $3(2,4]$ \\
Procedure & \\
Lobectomy & $43(86.0)$ \\
Wedge resection & $7(14.0)$ \\
\hline
\end{tabular}

Table 2 Preoperative surgical preparation and compliance

\begin{tabular}{lc}
\hline Preparation survey & No. $(\%)(\mathrm{n}=20)$ \\
\hline Received wound education & $17(85.0)$ \\
Print and verbal & $2(10.0)$ \\
Verbal only & $1(5.0)$ \\
Print only & \\
Received Hibiclens education & $16(80.0)$ \\
Print and verbal & $3(15.0)$ \\
Verbal only & $1(5.0)$ \\
None & $20(100.0)$ \\
NPO compliance & $20(100.0)$ \\
Hibiclens compliance & $13(65.0)$ \\
Compliance with exercise program (20-25 min/day) \\
Yes (all) \\
Yes (some) \\
No \\
Transportation difficulty
\end{tabular}

ambulation for the past day, and 18 (29\%) of reporting patients expressed worries about caring for themselves after discharge (Table 3).

\section{Postoperative bealth checks}

Postoperative health checks were completed by 27 (54\%) patients with a median of 3 [0-17] completed surveys per patient. The frequency of completed heath checks was highest during the first 14 days after surgery (17-22/day), but maintained at least 7 responses/day up to 30 days after surgery (Figure 2). Patients maximum rated pain level decreased gradually over the 30-day postoperative period $(\mathrm{P}=0.002)$. Similarly, patients self-reported anxiety level decreased significantly $(\mathrm{P}<0.001)$ after surgery, while mood level remained relatively stable ( $\mathrm{P}=0.06$; Figure 3$)$. Postoperative health checks were successful in improving patient's confidence in $23(85.2 \%)$ patients and decrease worries in $22(81.5 \%)$ patients. In addition, 9 patients $(40.9 \%)$ reported that the postoperative health checks through the App avoided at least one phone call to the care team, and $4(18.2 \%)$ patients reported they avoided one or more visit to the hospital (Table 3).

\section{Patient satisfaction}

The hospital satisfaction survey was completed by 31 (62\%) patients, of which $24(77.4 \%)$ gave the highest ranking ("excellent") for the care received, and 29 (93.5\%) reported they would recommend the hospital to others based on their experience.

Patients' perspectives in the use of the App are summarized in Table 4. Of 31 patients who responded to the preoperative and in-patient App feedback survey questions, 23 (74.2\%) reported the App was "very helpful" for preparing themselves for the operation, and "very" or "extremely useful" during their hospital stay. Patient's feedback regarding the usefulness of the App was similarly positive for the post-discharge recovery period, with twothirds of 33 patients who responded reporting they would "definitely recommend" the App (Table 4).

\section{Discussion}

This study evaluated the utility of a mobile patient engagement platform for PRO assessment in the perioperative period following minimally invasive robotic lung cancer surgery. Our results demonstrate high response 
Table 3 Postoperative patient reported experience and compliance

\begin{tabular}{|c|c|}
\hline Surveys & No. (\%) \\
\hline \multicolumn{2}{|l|}{ Inpatient recovery progress checks $(n=62)$} \\
\hline Uncontrolled pain & $9(14.5)$ \\
\hline \multicolumn{2}{|l|}{ Number of walks/day } \\
\hline 0 & $14(22.6)$ \\
\hline $1-2$ & $5(8.1)$ \\
\hline $3+$ & $43(69.4)$ \\
\hline \multicolumn{2}{|l|}{ Compliance with incentive spirometer use } \\
\hline Full compliance & $42(67.7)$ \\
\hline Some compliance & $18(29.0)$ \\
\hline No compliance & $2(3.2)$ \\
\hline Consumption of solid meals & $48(77.4)$ \\
\hline Worry about caring for self after discharge & $18(29.0)$ \\
\hline \multicolumn{2}{|l|}{ Inpatient satisfaction survey $(\mathrm{n}=31)$} \\
\hline \multicolumn{2}{|l|}{ Hospital care rating } \\
\hline Excellent & $24(77.4)$ \\
\hline Very good & $6(19.4)$ \\
\hline Good & $1(3.2)$ \\
\hline \multicolumn{2}{|l|}{ Would recommend this hospital } \\
\hline Definitely & $29(93.5)$ \\
\hline Probably & $2(6.5)$ \\
\hline \multicolumn{2}{|l|}{ Post-operative health checks $(n=27)$, median [IQR] } \\
\hline Number of health checks & $3[0-17]$ \\
\hline Times logged in & 7 [4-28] \\
\hline Mobile activated & $1[0-2]$ \\
\hline \multicolumn{2}{|l|}{ Calls made ( 30 days after leaving hospital) } \\
\hline 0 calls & $10(37.0)$ \\
\hline $1-2$ calls & $14(51.9)$ \\
\hline $3-4$ calls & $2(7.4)$ \\
\hline $5+$ calls & $1(3.7)$ \\
\hline \multicolumn{2}{|l|}{ Calls received ( 30 days after leaving hospital) } \\
\hline 0 calls & $15(55.6)$ \\
\hline $1-2$ calls & $7(25.9)$ \\
\hline $3-4$ calls & $2(7.4)$ \\
\hline $5+$ calls & $3(11.1)$ \\
\hline Health checks improved confidence & $23(85.2)$ \\
\hline Health checks decreased worries & $22(81.5)$ \\
\hline \multicolumn{2}{|l|}{ Calls avoided due to health checks $(n=22)$} \\
\hline 0 calls & $13(59.1)$ \\
\hline $1-2$ calls & $5(22.7)$ \\
\hline $3+$ calls & $4(18.2)$ \\
\hline $\begin{array}{l}\text { Hospital visits avoided due to health checks } \\
(n=22)\end{array}$ & $4(18.2)$ \\
\hline
\end{tabular}

rates of at least $40 \%$ amongst all surveys administered. Patient-reported compliance evaluations with instructions for presurgical preparation and in-hospital activities were overall good, but allowed for identification of several problem areas, such as adherence to preoperative exercise plans and inpatient activities, including spirometer use and mobility in the hospital. A large number of patients then remained engaged for up to two weeks after surgery, completing health checks and providing longitudinal symptom and quality-of-life data. In addition to providing the care team with real time PRO metrics, postoperative health checks through the App were able to increase the confidence and decrease worries in the majority of patients. Ultimately, a substantial number of patients reported that the use of the App prevented the need for phone calls or hospital visits.

Significant improvements have been made in the collection of PROs collection using electronic platforms. A recent systematic review by $\mathrm{Lu}$ and colleagues found a high satisfaction with automated electronic communication systems amongst patients and physicians in the perioperative setting (5). Specifically, mobile device applications have been proven useful in the perioperative setting in different surgical specialties, including breast, lung transplant, gastrointestinal, hepatobiliary and orthopedic surgery (4,6-10). A recent Cochrane review evaluated the literature on self-administered survey questionnaires by a mobile device App as compared to other traditional methods such the traditional paper questionnaires and concluded that delivery of surveys via Apps did not affect data equivalence and tended to improve the data completeness (11). Although the mobile App presents an innovative way for providers to approach patient care, there still exist some barriers related to its use. Considering the demographics of surgical lung cancer patients with a median age of over 60 , a number of barriers could affect patient's ability to access a smartphone or computer with internet. Participants from this age group come from a time when technology was not as big a part of their everyday lives and thus, they might be less astute with regards to the use of smartphones and mobile applications as compared to younger populations (12). Difficulties remembering and understanding how to use the App could cause frustration, a lack of compliance, and decreased satisfaction with the mobile platform. A study by Milne and colleagues found that although the self-perceived eHealth literacy amongst lung cancer survivors was generally low, approximately one third considered themselves to have high eHealth literacy, which was associated with the level of 


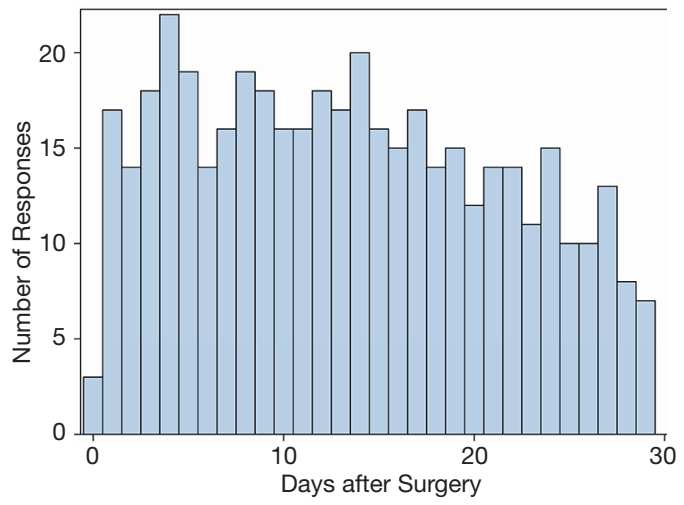

Figure 2 Frequency of completed postoperative health checks by postoperative day.
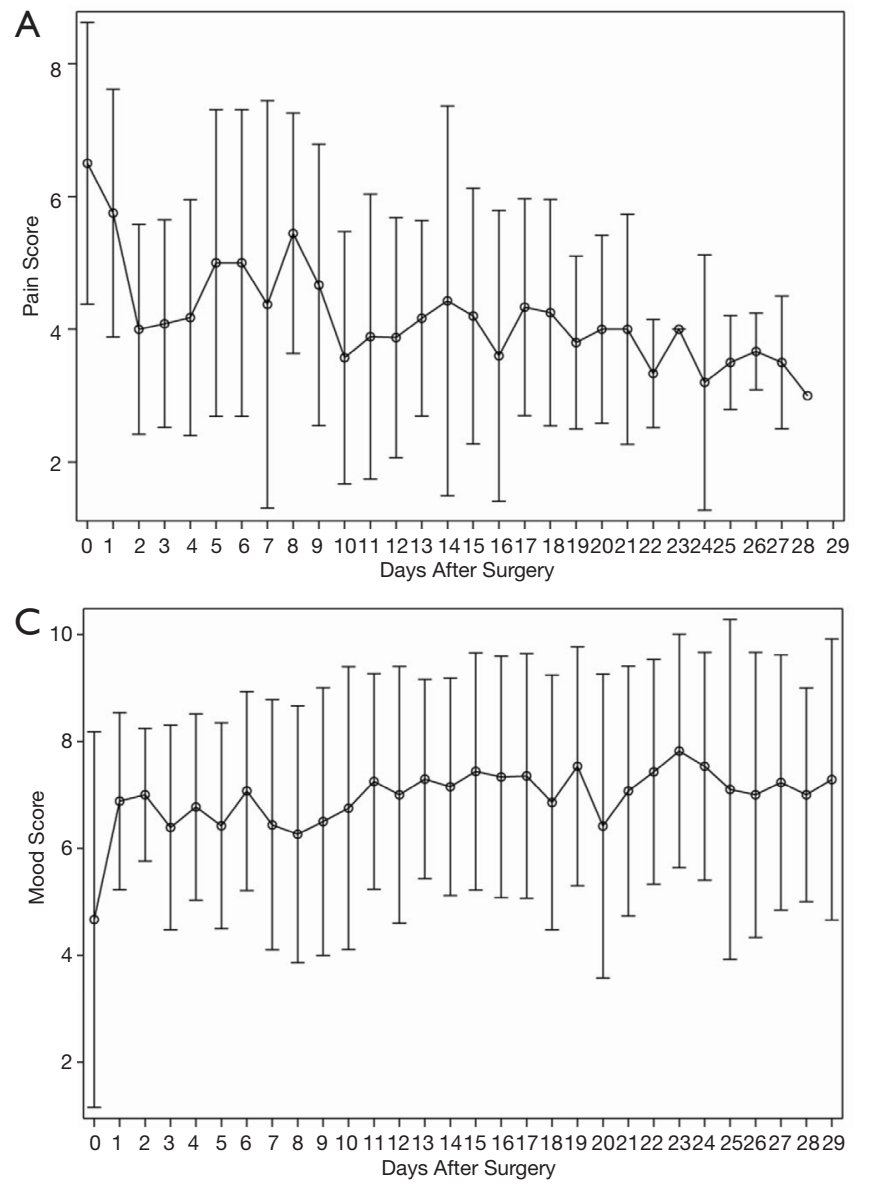

education and access to electronic resources (13). Additional barriers may exist, as for those patients living in rural areas, internet connection might be spotty and could thereby lead to frustration and a decreased desire to find ways to access and use an App. Some participants might also be unable to afford a smartphone and/or constant internet connection. This would prevent them from both participating in the study as well as in maintaining compliance. Additionally, patients might have access to the App through a family member (i.e., a daughter has a home with internet access) but might be limited in the consistency with which they can access it, thereby decreasing their potential compliance. Although we did not examine specific reasons of patients

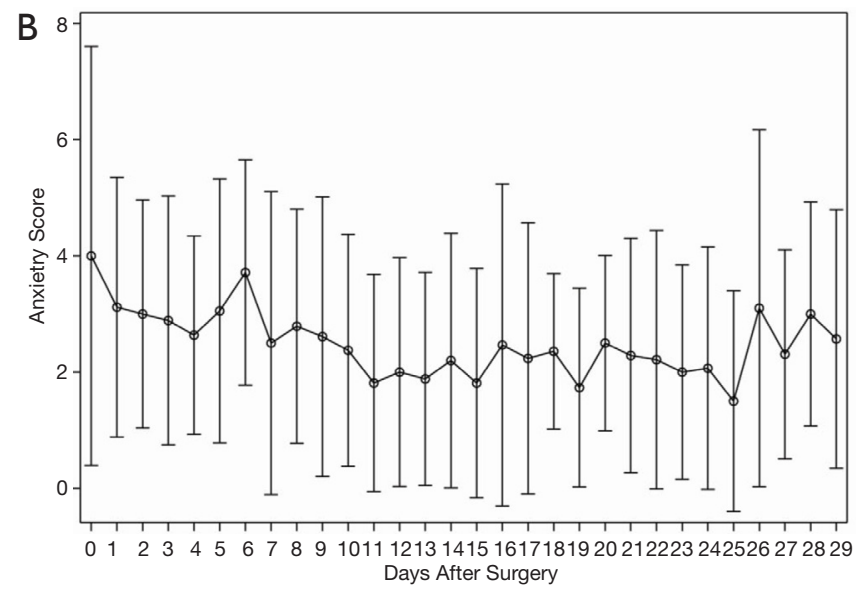

Figure 3 Postoperative trends of (A) maximum pain levels, (B) anxiety, and (C) mood levels during the 30-day postoperative period. Dots mark mean values and whiskers indicate standard errors. 
Table 4 Patient feedback regarding usefulness of the mobile device application (App)

\begin{tabular}{lc}
\hline Survey & No. (\%) \\
\hline Preoperative survey ( $\mathrm{n}=31)$ & \\
Surgery preparation facilitated by App & $23(74.2)$ \\
Very helpful & $8(25.8)$ \\
Little helpful & \\
In hospital survey ( $\mathrm{n}=31)$ & \\
App usefulness in hospital & $8(25.8)$ \\
Extremely useful & $15(48.4)$ \\
Very useful & $7(22.6)$ \\
Slightly useful & $1(3.2)$ \\
Not useful at all & \\
Post-discharge survey ( $\mathrm{n}=33)$ & \\
App usefulness after discharge & $11(33.3)$ \\
Extremely & $10(30.3)$ \\
Very & $16(48.5)$ \\
Slightly & $7(21.2)$ \\
Would recommend the App & \\
Definitely & \\
\hline
\end{tabular}

who were not able or did not elect to participate, our study found that despite all these hindrances, a large subset of patient was able to use the App. The highest use of the App in the first 14 days in surgical lung cancer patients in this study mirrors that of what has been shown for patients recovering from breast and orthopedic surgeries (10).

However, the self-reporting aspect of the mobile application provides benefits that might otherwise be difficult to obtain. The benefits of the examined App lies in the combination of patient engagement features and ability to track adherence to perioperative protocols with the collection of PROs (14). By relying $100 \%$ on patients to be compliant with post-operative protocols and treatment steps through the App, we are encouraging patients to take accountability for their health. The self-report measure, being optional, also supports patients in being honest with their responses. Knowing that the clarity and completeness of their responses could help further facilitate providers' care of them could also be a motivating factor for patients to be candid with their responses and promote self-management behaviors (6). Additionally, since the data is live and does not have to be audited by an abstracter, it allows surgeons real-time access to the information in order to make changes right away to a patient's treatment protocols (10). This allows the provider to impact outcomes in a positive manner almost immediately. Further, providers' abilities to respond quickly to patient concerns also encourages patients to view them favorably and likely increases their satisfaction with the post-operative process. The App also acts as a resource for patients. They have the ability to selfreport questions, concerns, and feedback on everything during the peri-operative period and provides an easy method by which they can feel heard by their healthcare team. Oftentimes, when patients have concerns regarding their health (e.g., whether or not the incision is infected), they may question the severity of the problem for a while before booking an appointment to meet with a provider by which point the problem might have worsened (15). Through the use of the application, patients have the opportunity to send pictures and receive instant clarification on their serious concerns. This also provides a chance for the doctor to maintain direct and open communication with their patients and facilitates the ability to immediately address problems. Previous studies have reported that such communication also reduces the number of phone and inperson encounters between the patient and the healthcare team $(10,16)$. Our study shows that patients themselves reported that they felt less of a need to make phone calls to the healthcare team with the use of the App following minimally invasive lung cancer surgery. This provides promise that the use of a mobile application could improve the efficiency by which a healthcare team is able to handle a patient's perioperative care.

Several limitations apply to this study. Although this project represents a novel way of gathering $\mathrm{PRO}$ and data on patient engagement and education in the lung cancer surgery patient population, it is limited by the size of the participant pool. The limited size of the patient participant pool also prevents us from making grand generalizations regarding the implications of the results of the study, which need to be confirmed in a larger scale randomized trial. Further, while there are a number of available mobile platforms our results are specific the Seamless $\mathrm{MD}^{\circledR}$ App and may not be generalizable. Other limitations to overcome in future projects include the lack of validation of survey questions in this study by a peer group or society for QOL and symptoms. The use of a health related QOL survey in the future would ensure that the questions are valid in 
testing general and disease specific PROs.

\section{Conclusions}

This study demonstrates that the use of a web-based application in the form of a mobile device App provides an efficient mechanism by which to record PROs and satisfaction with the peri-operative period while facilitating patient-provider engagement in post-surgical care. High patient satisfaction with mobile App-based management of perioperative care suggests that this method could be a positive step in bridging the gaps that might exist in provider-patient communications. Future studies are needed to assess the impact of patient engagement by mobile device applications on clinical outcomes such as readmission and complication rates.

\section{Acknowledgments}

The authors would like to thank Joshua Liu and the entire SeamlessMD team for their support with this pilot study. Funding: This work was supported by the following grants: Dr. D'Souza was supported by the OSU Wexner Medical Center Patient Safety Advancement Grant. Dr. Kneuertz was supported by the OSU Department of Surgery pilot study award.

\section{Footnote}

Provenance and Peer Review: This article was commissioned by the Guest Editor (Peter J. Kneuertz) for the series "Patient reported Outcomes in Thoracic Surgery: A new Frontier" published in Fournal of Thoracic Disease. The article has undergone external peer review.

Conflicts of Interest: All authors have completed the ICMJE uniform disclosure form (available at http://dx.doi. org/10.21037/jtd.2020.01.23). The series "Patient reported Outcomes in Thoracic Surgery: A new Frontier" was commissioned by the editorial office without any funding or sponsorship. PJK served as the unpaid Guest Editor of the series. REM reports personal fees from Intuitive Surgical, outside the submitted work. PJK reports grants from OSU Department of Surgery pilot study award, during the conduct of the study. The other authors have no other conflicts of interest to declare.

Ethical Statement: The authors are accountable for all aspects of the work in ensuring that questions related to the accuracy or integrity of any part of the work are appropriately investigated and resolved. The analysis of patient reported data was approved by the local Institutional Review Board (Buck-IRB-2019H0008) with waiver of informed consent. This study conformed to the provisions of the Declaration of Helsinki (as revised in 2013).

Open Access Statement: This is an Open Access article distributed in accordance with the Creative Commons Attribution-NonCommercial-NoDerivs 4.0 International License (CC BY-NC-ND 4.0), which permits the noncommercial replication and distribution of the article with the strict proviso that no changes or edits are made and the original work is properly cited (including links to both the formal publication through the relevant DOI and the license). See: https://creativecommons.org/licenses/by-nc-nd/4.0/.

\section{References}

1. Kneuertz PJ, Moffatt-Bruce SD. The Search for Meaningful Use of Patient Reported Outcomes in Thoracic Surgery. Ann Thorac Surg 2020;109:1317-8.

2. Singer ES, Merritt RE, D'Souza DM, et al. Patient Satisfaction After Lung Cancer Surgery: Do Clinical Outcomes Affect Hospital Consumer Assessment of Health Care Providers and Systems Scores? Ann Thorac Surg 2019;108:1656-63.

3. Chow A, Mayer EK, Darzi AW, et al. Patient-reported outcome measures: the importance of patient satisfaction in surgery. Surgery 2009;146:435-43.

4. Armstrong KA, Semple JL, Coyte PC. Replacing ambulatory surgical follow-up visits with mobile app home monitoring: modeling cost-effective scenarios. J Med Internet Res 2014;16:e213.

5. Lu K, Marino NE, Russell D, et al. Use of Short Message Service and Smartphone Applications in the Management of Surgical Patients: A Systematic Review. Telemed J E Health 2018;24:406-14.

6. DeVito Dabbs A, Song MK, Myers BA, et al. A Randomized Controlled Trial of a Mobile Health Intervention to Promote Self-Management After Lung Transplantation. Am J Transplant 2016;16:2172-80.

7. Gustavell T, Langius-Eklöf A, Wengström Y, et al. Development and Feasibility of an Interactive Smartphone App for Early Assessment and Management of Symptoms Following Pancreaticoduodenectomy. Cancer Nurs 2019;42:E1-10. 
8. Kim CH, Chung CK, Choi Y, et al. The usefulness of a mobile device-based system for patient-reported outcomes in a spine outpatient clinic. Spine J 2016;16:843-50.

9. Malhotra K, Buraimoh O, Thornton J, et al. Electronic capture of patient-reported and clinician-reported outcome measures in an elective orthopaedic setting: a retrospective cohort analysis. BMJ Open 2016;6:e011975.

10. Semple JL, Sharpe S, Murnaghan ML, et al. Using a mobile app for monitoring post-operative quality of recovery of patients at home: a feasibility study. JMIR Mhealth Uhealth 2015;3:e18.

11. Marcano Belisario JS, Jamsek J, et al. Comparison of selfadministered survey questionnaire responses collected using mobile apps versus other methods. Cochrane Database Syst Rev 2015;(7):MR000042.

12. Tang MY, Li ZC, Dai Y, et al. What Kind Of A Mobile Health App Do Patients Truly Want? A Pilot Study Among Ambulatory Surgery Patients. Patient Prefer

Cite this article as: Kneuertz PJ, Jagadesh N, Perkins A, Fitzgerald M, Moffatt-Bruce SD, Merritt RE, D'Souza DM. Improving patient engagement, adherence, and satisfaction in lung cancer surgery with implementation of a mobile device platform for patient reported outcomes. J Thorac Dis 2020;12(11):6883-6891. doi: 10.21037/jtd.2020.01.23
Adherence 2019;13:2039-46.

13. Milne RA, Puts MT, Papadakos J, et al. Predictors of High eHealth Literacy in Primary Lung Cancer Survivors. J Cancer Educ 2015;30:685-92.

14. Pecorelli N, Fiore JF Jr, Kaneva P, et al. An app for patient education and self-audit within an enhanced recovery program for bowel surgery: a pilot study assessing validity and usability. Surg Endosc 2018;32:2263-73.

15. Jaensson M, Dahlberg K, Eriksson M, et al. The Development of the Recovery Assessments by Phone Points (RAPP): A Mobile Phone App for Postoperative Recovery Monitoring and Assessment. JMIR Mhealth Uhealth 2015;3:e86.

16. Armstrong KA, Coyte PC, Brown M, et al. Effect of Home Monitoring via Mobile App on the Number of In-Person Visits Following Ambulatory Surgery: A Randomized Clinical Trial. JAMA Surg 2017;152:622-7. 